\title{
Local influence of magnetosheath plasma beta fluctuations on magnetopause reconnection
}

\author{
T. V. Laitinen ${ }^{1, *}$, Y. V. Khotyaintsev ${ }^{1}$, M. André ${ }^{1}$, A. Vaivads ${ }^{1}$, and H. Rème ${ }^{2,3}$ \\ ${ }^{1}$ Swedish Institute for Space Physics, P.O. Box 537, 75121 Uppsala, Sweden \\ ${ }^{2}$ University of Toulouse, UPS, CESR, 9 avenue colonel Roche, 31028 Toulouse cedex 4, France \\ ${ }^{3}$ CNRS, UMR5187, 31028 Toulouse, France \\ * now at: Finnish Meteorological Institute, PL 503, 00101 Helsinki, Finland
}

Received: 11 May 2009 - Revised: 22 January 2010 - Accepted: 13 April 2010 - Published: 5 May 2010

\begin{abstract}
We present observations from two subsolar Cluster magnetopause crossings under southward interplanetary magnetic field and strong mirror mode fluctuations in the magnetosheath. In both events the reconnection outflow jets show strong variations on the timescale of one minute. We show that at least some of the recorded variations are truly temporal, not spatial. On the same timescale, mirror mode fluctuations appear as strong magnetic fluctuations in the magnetosheath next to the magnetopause. This suggests that mirror modes can cause the variations either through modulation of continuous reconnection or through triggering of bursty reconnection. Using a theoretical scaling law for asymmetric reconnection we show that modulation of reconnection at a single $\mathrm{x}$-line can explain the observations of the first event. The second event cannot be explained by a single modulated $\mathrm{x}$-line: there the evidence points to patchy and bursty reconnection.
\end{abstract}

Keywords. Magnetospheric physics (Magnetopause, cusp, and boundary layers) - Space plasma physics (Magnetic reconnection)

\section{Introduction}

The location and dynamics of reconnection on the magnetopause is one of the most important factors governing magnetospheric dynamics. The traditional view on the process derives from the Dungey (1961) convection cycle: during southward interplanetary magnetic field (IMF), magnetopause reconnection occurs on a global separator line which

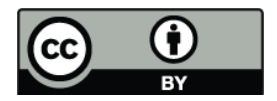

Correspondence to: $\mathrm{T}$. V. Laitinen (tiera.laitinen@fmi.fi) extends across the dayside magnetopause. Observations have confirmed that such a long reconnection line exists at times (e.g. Phan et al., 2006), and it is also reproduced by global MHD simulations (e.g. Laitinen et al., 2006, 2007). Lately much attention has been given to determining the location of the reconnection line under different IMF orientations (e.g. Lockwood et al., 2003; Trattner et al., 2007). The two competing hypotheses, component reconnection and antiparallel reconnection, both view reconnection as occurring continuously in time and at one smooth large-scale reconnection line (component hypothesis predicts the $\mathrm{x}$-line to be continuous in space, while antiparallel hypothesis predicts one discontinuity at the subsolar region).

On the other hand, we also know that bursty and spatially localised reconnection can occur at the magnetopause. Flux transfer events (FTE) are its common observational signature (Russell and Elphic, 1979). They are observed during all kinds of solar wind and IMF conditions, with certain statistical trends, and at temporal separations ranging from less than a minute upwards (Wang et al., 2006). The exact nature of FTE-producing reconnection events is uncertain. Ku and Sibeck (1998) concluded from their 2-dimensional MHD simulations that bursty merging along a single extended $\mathrm{x}$ line does not explain the symmetric bipolar signature of a typical FTE. As alternatives, they suggest bursty merging at a point or along at least two parallel $\mathrm{x}$-lines. Bursty reconnection at a point - or on a short piece of $\mathrm{x}$-line - is supported by the longitudinally limited spatial size of the FTE structure, inferred by Kawano and Russell (2005) from observed FTE motions.

What determines when magnetopause reconnection is steady and when it is bursty, patchy or otherwise non-steady? Is it ever really steady, if examined at good enough resolution, or is steady reconnection only the big-scale average

Published by Copernicus Publications on behalf of the European Geosciences Union. 


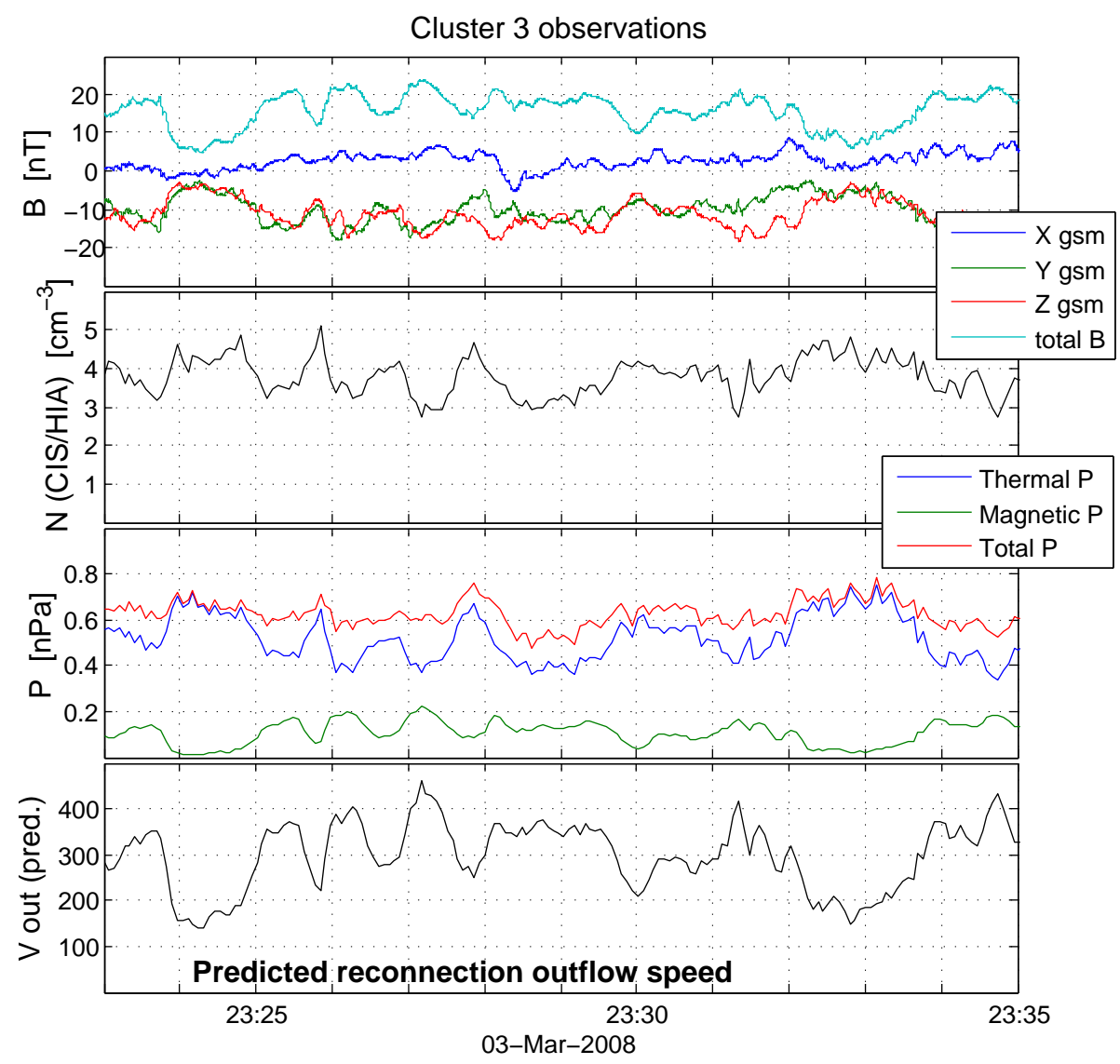

Fig. 1. Mirror modes in Cluster 3 observations, slightly after event 2. The first panel shows magnetic field components in GSM coordinates and the total magnetic field (light blue). In second panel, ion density. Third panel: plasma thermal pressure, magnetic pressure and their sum. The sum stays approximately constant, a typical property of mirror modes. Fourth panel shows the reconnection outflow velocity calculated by Eq. (2), using observed magnetosheath values from the first two panels and a constant value of $B_{1}=28 \mathrm{nT}$ for the magnetosphere magnetic field.

effect of non-steady small-scale processes? Having analysed in detail one FTE and concluded that it was generated by component merging, Sonnerup et al. (2004) pose the following questions: "Does component merging by necessity occur in a bursty fashion, leading to the generation of FTEs, or is the bursty behavior controlled by factors other than, or in addition to, the presence of a guide field, i.e. a magnetic field component in the expected direction of the x-line? Does quasi-steady reconnection imply anti-parallel fields and/or vice versa?"

When studying the properties of magnetopause reconnection, the relevant boundary conditions are the magnetic field orientation and the plasma properties in the magnetosheath. The magnetic field direction is affected by draping of the field lines: according to Coleman (2005), the IMF clock angle is conserved within $30^{\circ}$ in about $70 \%$ of cases, and in about $90 \%$ of cases in the subsolar region. Compression of plasma at the bow shock is obvious, but less obvious is the effect of different oscillations and fluctuations in the mag- netosheath. One type of possibly significant fluctuations are variations of plasma beta, which can be caused by e.g. mirror modes.

Mirror mode fluctuations are commonly observed in the terrestrial magnetosheath (e.g. Tsurutani et al., 1982; Lucek et al., 2001; Tátrallyay and Erdős, 2002; Soucek et al., 2008), and also in the solar wind and in magnetosheaths and magnetospheres of other planets (e.g. Treumann et al., 2004; Joy et al., 2006). They are co-moving compressional nonoscillatory magnetic variations which exhibit local anticorrelation of magnetic and thermal pressures, suggesting that they are in local pressure equlibrium, and do not involve significant variations in magnetic field direction. Some mirror modes in the magnetosheath, of the type that were prevalent during the events that we discuss in this paper, are shown in Fig. 1. In this case the mirror modes form a quasi-sinusoidal pattern, but they are also rather frequently observed as isolated large magnetic peaks or holes (Joy et al., 2006; Soucek et al., 2008; Génot et al., 2009). Treumann et al. (2004) 
describe mirror modes as long magnetic field-depleted tubes. The amplitude of the magnetic field variation often exceeds $50 \%$ of the background field. In the transverse direction their typical size in the magnetosheath is several hundreds of kilometers, corresponding to a few ion gyroradii (Lucek et al., 2001). Mirror modes are generated by the mirror instability in anisotropic plasma where the mirror instability condition is satisfied, $\beta_{\perp}\left(T_{\perp} / T_{\|}-1\right)<1$ (Hasegawa, 1969). Once generated they may survive even where the condition is not satisfied (Soucek et al., 2008; Génot et al., 2009). Therefore the mirror instability condition is a necessary condition for the triggering of mirror modes but not for their presence.

In this paper we present Cluster observations on two events of non-steady magnetopause reconnection. One common feature of our events is the presence of strong plasma beta fluctuations in the magnetosheath. While the origin of such fluctuations is not important in the limited scope of this paper, we identify them as mirror mode fluctuations to make the treatment more concrete and to point out that the mirror instability also plays a role in solar wind-magnetosphere coupling.

\section{Plasma beta influence on reconnection: theoretical motivation}

In mirror mode fluctuations the magnetic field strenth $B_{2}$ and the density $\rho_{2}$ always change in antiphase. As mirror modes are larger than the ion gyroradius, it is not unrealistic to approximate them as spatially large compared to the size of the reconnection diffusion region. Thus an incoming mirror mode can be considered as a temporary change of the plasma beta in the inflowing plasma on one side of the diffusion region. Consider a mirror mode with reduced magnetic field and enhanced plasma density. When it drifts into the reconnection inflow region, the magnetic energy available for acceleration per unit mass of plasma is reduced. Reduction of outflow velocity can thus be expected.

The outflow velocity is commonly expected to be of the order of magnitude of the Alfvén speed in the inflow region. This scaling law was generalised for asymmetric inflow regions by Cassak and Shay (2007): the outflow velocity is expected to scale as

$v_{\text {out }}^{2} \sim \frac{B_{1} B_{2}}{\mu_{0}} \frac{B_{1}+B_{2}}{\rho_{1} B_{2}+\rho_{2} B_{1}}$,

where subscripts 1 and 2 refer to the two inflow regions (note that the expression is invariant under an interchange of 1 and $2), B$ is the magnetic flux density and $\rho$ is the plasma mass density. If we let 1 denote the magnetosphere and 2 denote the magnetosheath at the subsolar magnetopause, we have $\rho_{1} B_{2} \ll \rho_{2} B_{1}$, and we may simplify the expression as

$v_{\text {out }}^{2} \sim \frac{B_{2}}{\mu_{0} \rho_{2}}\left(B_{1}+B_{2}\right)$.
We see immediately from Eq. (2) that a reduction in outflow velocity is to be expected whenever the magnetic flux density is reduced. The lowest panel in Fig. 1 illustrates how the modulated outflow velocity could behave. It is a direct application of Eq. (2) to the observational data. The expected outflow would consist of faster jets separated by intervals of slower exhaust. The timescale of these outflow speed variations is set by the timescale of the incoming magnetic variations. As mirror modes are co-moving, this timescale is simply their spatial scale size divided by the average inflow speed to the reconnection region. In the case shown in Fig. 1 this timescale is of the order of one minute. On a global magnetopause reconnection line this modulation would be local, as the size of mirror modes is small compared to the size of the magnetosphere.

\section{Observations}

We examine data collected by several instruments onboard the Cluster spacecraft (Escoubet et al., 2001): magnetic field from FGM (Balogh et al., 2001), ion velocity and density from CIS/HIA (Rème et al., 2001), and density estimates derived from the spacecraft potential measured by EFW (Pedersen et al., 2008). As CIS/HIA data is only available from Cluster 1 and 3 during the times of interest, we use mostly data from these two satellites. We selected events with the following criteria:

- Magnetopause crossing near the subsolar point (Cluster orbital parameters thus limiting the search to 2007 and 2008 spring seasons)

- Stable enough magnetosheath conditions with approximately constant $\boldsymbol{B}$ direction

- Southward $\boldsymbol{B}$ in the magnetosheath

- Reconnection jet recorded at a magnetopause crossing

- Prolonged stay of at least several minutes at or near the magnetopause, i.e. observation not limited to one quick crossing.

Having evaluated the 2007 and 2008 spring season magnetopause crossings, we present observations from the two events that best fulfil these criteria.

\subsection{Event 1: 27 March 2007}

On 27 March 2007 the Cluster quartet crossed the magnetopause at $(X, Y, Z)=(9,0,3.5) R_{\mathrm{E}}$ in GSE coordinates, i.e. slightly north of the subsolar point. The relative positions of the spacecraft are shown in Fig. 2. To analyse the event, we use boundary normal coordinates $\{L, M, N\}$ defined as follows. $L$ is the direction of magnetic field in the magnetosphere (averaged over an interval of about $10 \mathrm{~min}$ 


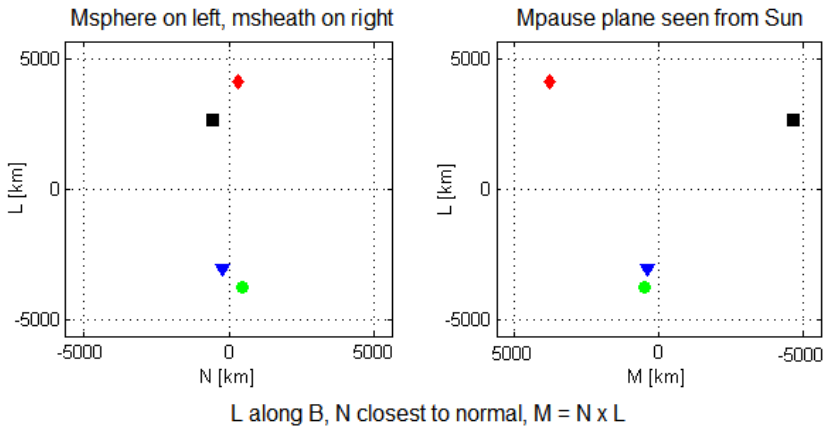

Fig. 2. Relative positions of the Cluster satellites during event 1 , on 27 March 2007 at 05:10 UT. The figure is drawn in boundary normal coordinates (see text for definition), where $L$ is close to GSE $Z$ and $N$ close to GSE $X$. The origin of the plot is at $(9,0,3.5) \mathrm{R}_{\mathrm{E}}$ in GSE. $\mathrm{C} 1$ is black square, $\mathrm{C} 2$ red diamond, $\mathrm{C} 3$ green circle and $\mathrm{C} 4$ blue triangle.

before crossing), $N$ is the direction closest to the magnetopause normal in the Tsyganenko 95 model (Tsyganenko, 1995) and perpendicular to $L$, and $M=N \times L$. This leads to $L=(-0.29,0.39,0.87), M=(-0.13,-0.92,0.37)$, and $N=(0.95,-0.007,0.32)$ in GSE. The actual orientation of the magnetopause current sheet during different crossings is not relevant to this study, and therefore we do not use minimum variance analysis to determine it. Rather, the Tsyganenko model gives a simple approximation of the average orientation of the magnetopause. This is useful for adjusting the coordinate system to the large-scale reconnection geometry and allows us to use the same coordinate system throughout the half an hour long interval with several magnetopause crossings.

Figure 3 shows the magnetic field, ion velocity and ion density at Cluster satellites 1 and 3, henceforth referred to as $\mathrm{C} 1$ and $\mathrm{C} 3$. $\mathrm{C} 1$ moves from the magnetosphere to the magnetosheath at about 05:07 UT. The angle between mean magnetic field direction inside and outside the magnetopause is about $160^{\circ}$, so the fields are almost antiparallel. Northward (positive $L$ ) oriented plasma jets are observed on both spacecraft during the interval 05:04-05:17. Their speed exceeds $300 \mathrm{~km} \mathrm{~s}^{-1}$ at several instances, the fastest jet reaching almost $500 \mathrm{~km} \mathrm{~s}^{-1}$. This jet is shown at higher time resolution in Fig. 4.

The data in Fig. 3 also show that the magnetosheath is full of mirror modes. Mirror mode magnetic signatures can be seen whenever the spacecraft are in the magnetosheath (which is recognised by negative $B_{L}$ values, while in the magnetosphere $B_{L} \sim+70 \mathrm{nT}$ ). They appear as quasi-periodic magnetic depressions with a typical amplitude $\Delta B / B \sim 50 \%$, and for the strongest fluctuations the amplitude reaches $80 \%$. The fluctuations are approximately linearly polarized, as is characteristic for mirror modes: the associated change in magnetic field direction is less than $50^{\circ}$ for most of the fluctuations. The expected anticorrelation of magnetic field magnitude and plasma density is not always distinguishable due to the time resolution of the ion data, but it can be seen for the most representative mirror modes. Best examples are $\mathrm{C} 1$ at 05:12-05:13:30 and $\mathrm{C} 3$ at 05:09-05:10. The plasma temperature anisotropy (not shown) also satisfies the mirror instability condition (Hasegawa, 1969).

One way to predict the tilted $\mathrm{x}$-line location is to integrate the line starting from the subsolar point so that the tangential direction of the line at each point is given by the condition that the antiparallel components of the merging magnetic fields are maximized (Trattner et al., 2007). Taking the magnetospheric magnetic field direction from the Tsyganenko 96 model and the magnetosheath field direction from a draping model, they predicted the $\mathrm{x}$-line to be located about $3 R_{\mathrm{E}}$ south of the $\mathrm{C} 1$ location for this event $(\mathrm{K}$. Trattner, private communication). The observed ambient flow speed in the magnetosheath is $100-150 \mathrm{~km} \mathrm{~s}^{-1}$. The ambient flow and the jets are both directed northward (positive $L$ ), as expected since the satellites are located north of the subsolar point and of the predicted $\mathrm{x}$-line location. The jet velocities also roughly agree with what Eq. (1) predicts. Thus reconnection at the subsolar $\mathrm{x}$-line is a likely source for the observed plasma jets.

We also performed a Walén test (see Khrabrov and Sonnerup, 1998) for the outbound magnetopause crossing after the fast jet in Fig. 4, 05:07:40-05:13:05 UT. The deHoffmanTeller (HT) frame was found to be moving at a speed of $v_{\mathrm{HT}}=268 \mathrm{~km} \mathrm{~s}^{-1}$ with respect to the GSE frame. Figure 5 is the Walén scatter plot for the interval. It shows the correlation between the plasma flow speed in the HT frame and the local Alfvén speed. The correlation coefficient is extremely good, 0.98 , indicating that the HT frame determination is good and the test result relevant. The coefficient of proportionality is 0.72 . This is reasonably close to unity, indicating that the magnetopause has the structure of a large-amplitude Alfvén wave or rotational discontinuity, as expected in the vicinity of a reconnection site. (In contrast, a coefficient of proportionality close to zero would point to the magnetopause being a tangential discontinuity, which would prove against reconnection.) The insert in the figure shows the ion distribution function during the jet, with the velocity component parallel to the magnetic field on the vertical axis. The highest velocity space density, represented by red colour, forms a D-shaped distribution charasteristic of reconnection jets (Cowley, 1982). The distribution has a cutoff at the HT frame speed as expected. This evidence strongly supports the interpretation of the observed fast plasma flows as reconnection jets.

At 05:05-05:08, C3 stays near the magnetopause for three minutes, as indicated by the rapid variation of the magnetic field $L$ component between values typical of the magnetosphere and the magnetosheath. During this time, the satellite sees an almost continuous fast flow, whose speed, however, varies rapidly. Otherwise all jet occurrencies during the 


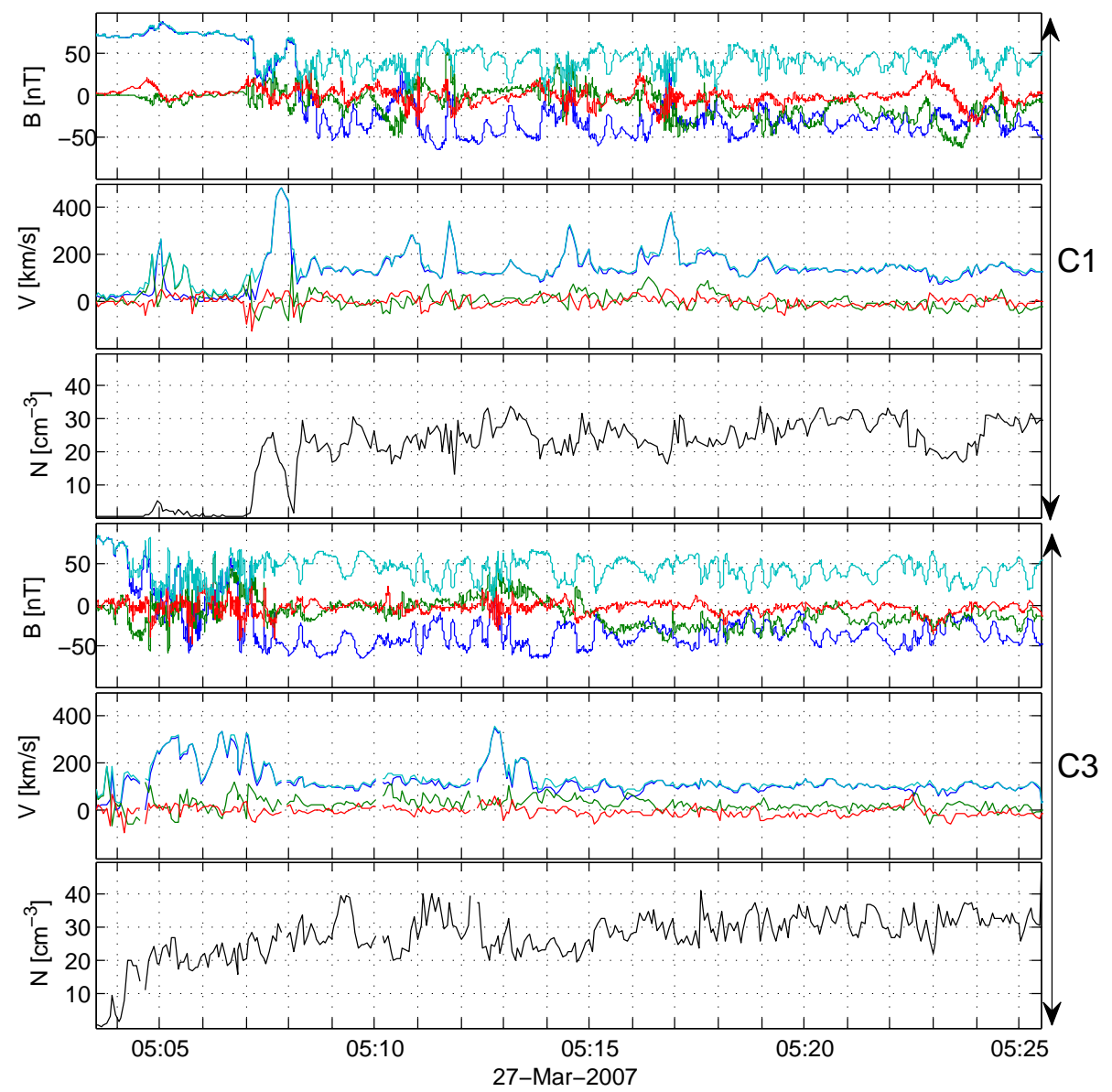

Fig. 3. Magnetic field, ion velocity and ion density recorded by Cluster 1 and 3 during event 1, on 27 March 2007 at 05:04-05:25 UT at the magnetopause approximately $4 \mathrm{R}_{\mathrm{E}}$ north from the subsolar point. For $\boldsymbol{B}$ and $\boldsymbol{V}$, light blue curve gives the absolute magnitude. Dark blue, green and red are $L, M$ and $N$ components, respectively, in boundary normal coordinates defined in the text.

interval last about half a minute. Half a minute is also the timescale of the mirror mode fluctuations as they advect past the spacecraft.

The separation between the different Cluster satellites is large, about $1 R_{\mathrm{E}}$, and they are not aligned with the plasma jets, so that in general we do not record the same jet at several spacecraft. These are thus essentially single-spacecraft observations, and that makes it difficult to discriminate between temporal and spatial variations. In most instances, the recorded changes in flow speed could be due to either true changes in the reconnection jet speed or movement of the magnetopause and jet with respect to the satellite. However, in one case we are able to identify the variation as temporal.

The jet where temporal changes can be established is the one recorded by $\mathrm{C} 1$ at 05:07:30-05:08 UT, shown in Fig. 4. $\mathrm{C} 1$ crosses the magnetopause halfway at 05:07:10 and, judging from $B_{L}$, is farthest away from the magnetosphere at 05:07:23. (We use $B_{L}$ to indicate where the satellite is between the magnetosphere and the magnetosheath, cf. Fig. 6.) Until this time the plasma flow speed is under $200 \mathrm{~km} \mathrm{~s}^{-1}$. Around 05:07:40, while $B_{L}$ at the location of $\mathrm{C} 1$ is slowly increasing, the plasma flow speed increases to almost $500 \mathrm{~km} \mathrm{~s}^{-1}$. Then at 05:08:00 $\mathrm{C} 1$ enters the magnetosphere again and the measured flow velocity drops to $200 \mathrm{~km} \mathrm{~s}^{-1}$. This might be due to $\mathrm{C} 1$ moving deeper into the magnetosphere, out of the jet at the magnetopause, but only $10 \mathrm{~s}$ later $\mathrm{C} 1$ crosses the magnetopause again, and sees no accelerated plasma flow. We should see the jet at all three crossings, if the reconnection jet at the magnetopause were continuous. Thus we conclude that the duration of this fast flow was less than the interval between the first and third magnetopause crossing in Fig. 4, i.e. less than 60 s.

The variability of the outflow speed is further illustrated in Fig. 6, where the jet speed is plotted against the magnetic field $L$ component. Data points from the magnetosphere appear as a dense cluster in the lower right corner, while data from the magnetosheath forms an elongated cluster at the lower left (elongation is due to the mirror modes). The 


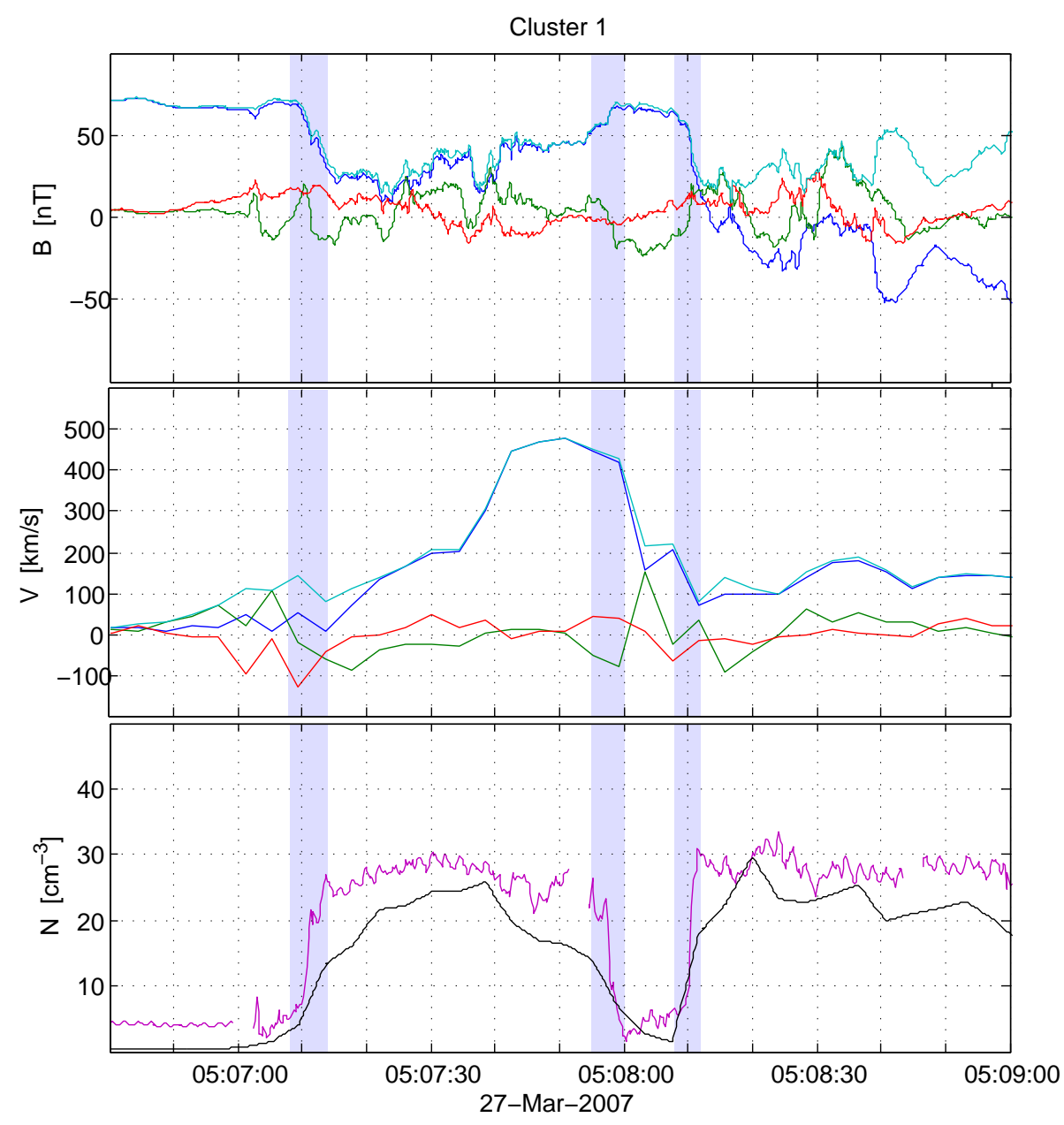

Fig. 4. A detail from Fig. 3: Cluster 1 observation at 05:07:40-05:09:00 UT. $\boldsymbol{B}$ and $\boldsymbol{V}$ are in same format as in Fig. 3. In the ion density panel, the black curve is the CIS/HIA ion measurement (same as in Fig. 3) and the magenta curve is density estimate derived from the EFW spacecraft potential measurement, added here because it has higher time resolution. The 4-s oscillation is due to spacecraft spin. Blue vertical bars mark the three partial magnetopause crossings during this interval. If reconnection at the magnetopause were continuous and steady, the reconnection jet should be observed at all three crossings.

scattered points in between are measurements during transitions through the magnetopause. The highest jet speeds, corresponding to the jet in Fig. 4 , are recorded at $B_{L}=$ $40-60 \mathrm{nT}$, but also lower plasma flow speeds, down to the ambient magnetosheath flow speed, are recorded in the same $B_{L}$ interval. In fact, the scatter plot shows instances of both accelerated and non-accelerated plasma flow everywhere in the layer between the magnetosphere and the magnetosheath.

At 05:23 UT we also see a clear bipolar variation in the normal component of the magnetic field at $\mathrm{C} 1$ (Fig. 3), indicating the passage of an FTE-like structure. Other similar variations with the same polarity are recorded by $\mathrm{C} 1$, such as those at 05:05 and 05:16. This is further evidence for nonsteady reconnection.

We conclude that in event 1 nearly antiparallel reconnection at the subsolar magnetopause was quasi-continuous. A reconnection jet was observed most of the times when a spacecraft approached the magnetopause, but the jet speed varied from $500 \mathrm{~km} \mathrm{~s}^{-1}$ down to the ambient magnetoheath flow speed, and at least in one case the accelerated plasma flow lasted less than $60 \mathrm{~s}$. The timescale of the variations is roughly half a minute, the same as the timescale of the mirror mode fluctuations observed immediately outside the magnetopause.

\subsection{Event 2: 3 March 2008}

Our second event occurred on 3 March 2008, 23:0523:20 UT. For this event we use the GSM coordinate system, as it happens to be virtually identical to the boundary normal system, GSM $X$ corresponding to the magnetopause normal and GSM $Z$ to the magnetic field direction 


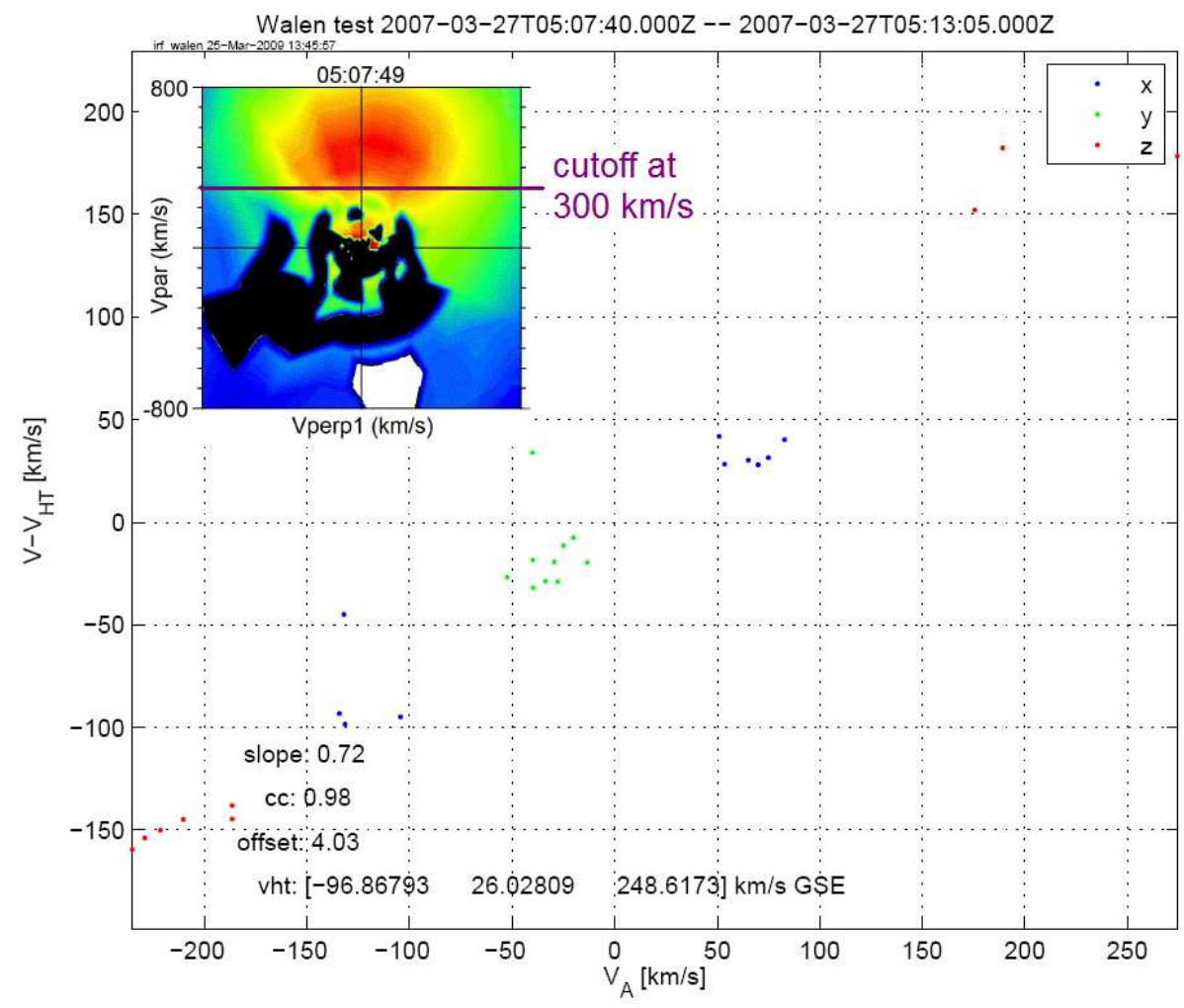

Fig. 5. Walén test for the outbound magnetopause crossing immediately after the observation of the fast jet in Fig. 4 . The insert shows a sample ion distribution function during the fast jet: The D-shaped jet population, advancing parallel to the magnetic field, has a lower cutoff at the HT frame speed.

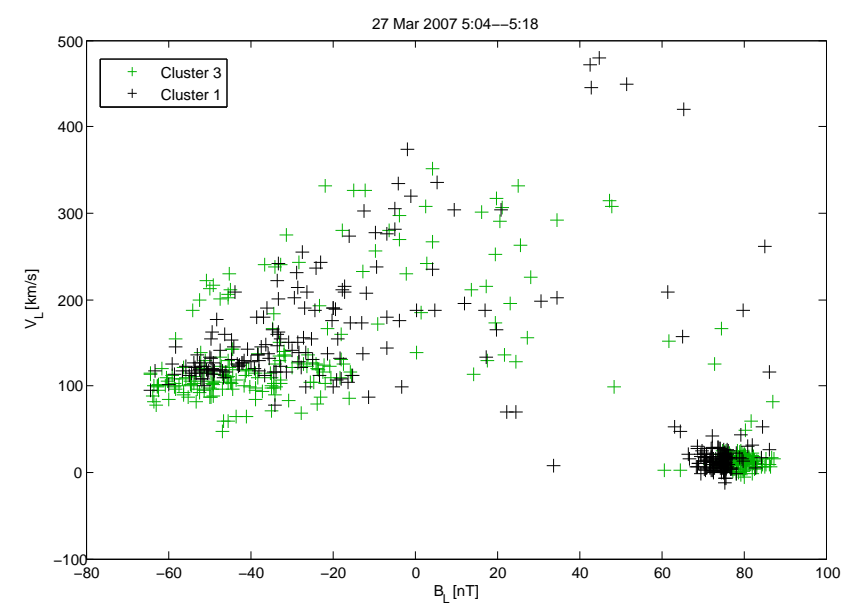

Fig. 6. Scatter plot of $V_{L}$ versus $B_{L}$ in event 1, during 05:0405:25 UT (same interval as plotted in Fig. 3). Black crosses are data points from $\mathrm{C} 1$ and green crosses from $\mathrm{C} 3$. Both low and high flow speeds are recorded at all $B_{L}$ values between the magnetosphere and the magnetosheath, indicating that no stable plasma jet exists between the regions.
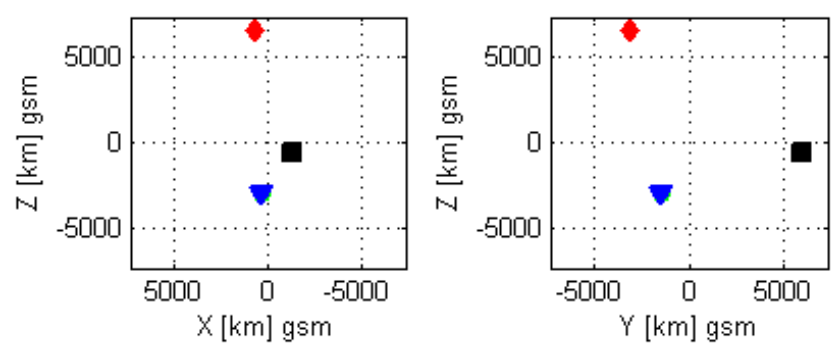

Fig. 7. Relative positions of the Cluster satellites during event 2, on 3 March 2008 at 23:15 UT. The figure is drawn in GSM coordinates. $\mathrm{C} 1$ is black square, $\mathrm{C} 2$ red diamond, $\mathrm{C} 3$ green circle (under the $\mathrm{C} 4$ symbol) and C4 blue triangle.

in the magnetosphere. The location of the crossing was $(X, Y, Z)=(13.5,3.4,2.1) R_{\mathrm{E}} \mathrm{GSM}$, i.e. slightly duskward from the subsolar point. The satellite configuration is shown in Fig. 7 and the observations in Fig. 8.

C3 sees the magnetopause for the first time around 23:06 UT, C1 around 23:09, and after skimming the magnetopause a few times, both spacecraft finally pass from the magnetosphere into the magnetosheath around 23:19. The 


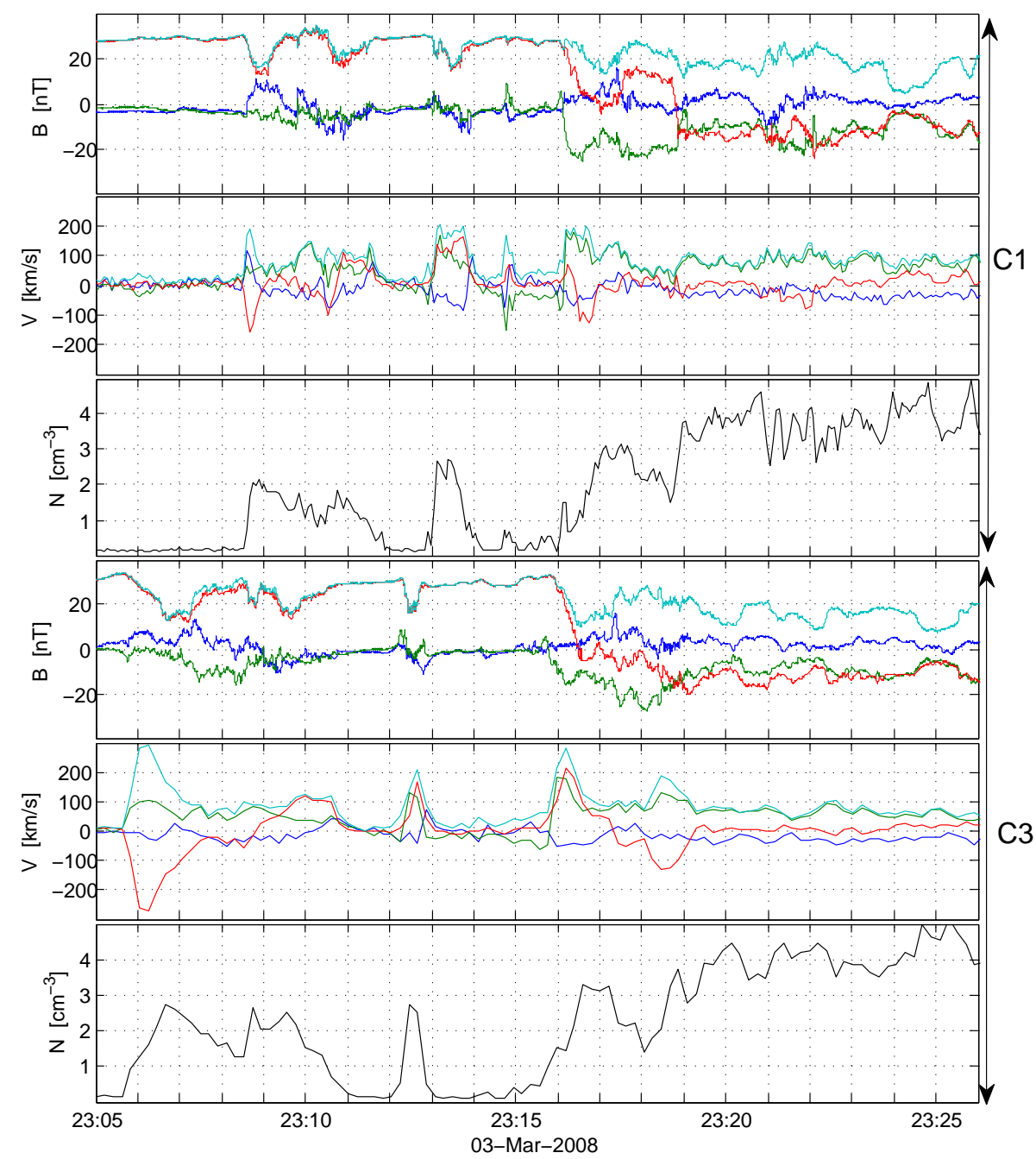

Fig. 8. Magnetic field, ion velocity and ion density during event 2, on 3 March 2008 at 23:05-23:26 UT at the magnetopause slightly duskward and northward from the subsolar point. Data is shown in GSM coordinates. For $\boldsymbol{B}$ and $\boldsymbol{V}$, light blue curve gives the absolute magnitude. Dark blue, green and red are $X, Y$ and $Z$ components, respectively.

angle between the mean magnetic field direction inside and outside the magnetopause at $\mathrm{C} 3$ is about $140^{\circ}$, so the fields have large antiparallel components but there is also a guide field. The magnetosheath is again densely populated with mirror modes. They are identified by the same characteristics as in event 1 , the variation in magnetic field direction being slightly smaller $\left(\lesssim 30^{\circ}\right)$. One of them is visible in C1 data at 23:24 and several others in C3 data at 23:20-23:26, more mirror modes from this event are seen in Fig. 1.

As in event 1, numerous plasma jets with duration on the order of one minute are seen. The special feature of this event is that the spacecraft also record several flow reversals: $\mathrm{C} 1$ at 23:10:40 and 23:16:30, C3 at roughly 23:08 and 23:17:30. The flow directions and velocities at $\mathrm{C} 1$ and $\mathrm{C} 3$, separated by about $8000 \mathrm{~km}$, seem rather uncorrelated. The only common general feature is that the jets at both satellites tend to have a duskward velocity component, which is natural, as the satellites are duskward of the subsolar point and the ambient flow in the sheath is also in that direction.

The predicted location of the global scale magnetopause reconnection line coincides with the $\mathrm{C} 3$ location (K. Trattner, private communication). As the jets in this event are distinguished from the ambient flow even by their direction and show a temporal variation similar to that in event 1 , we judge it likely that they are reconnection outflows. This is supported by the observation of density cavities in conjunction with some of the beginnings or endings of jets (not visible in HIA data, but similar cavities in event 1 are seen at 05:07:04 and 05:08:00 in EFW density data in Fig. 4.). Such cavities are part of the structure of reconnection separatrix regions (Retinò et al., 2006). The slope in the Walén test was close to zero, which indicates that the magnetopause was a 
tangential discontinuity rather than a rotational one. This testifies against continuous large-scale reconnection, but is compatible with sporadic and localised reconnection, without large-scale $\mathrm{x}$-line, and not very close to the observing spacecraft. Next we argue for this interpretation.

Different outflow directions at different locations are seen in Fig. 8 e.g. at 23:10 and 23:16. This suggests that the flows are generated by patchy reconnection on a scale larger than the inter-spacecraft separation. Flow reversals $(\mathrm{C} 1$ at 23:10:40 and 23:16:20. C3 at 23:08 and 23:17:30) at one spacecraft might also be a signature of an $\mathrm{x}$-line moving past the satellite, but the different flow directions at different locations simultaneously make different short-lived reconnection sites a more likely explanation.

An exception to the lack of correlation between the jets at $\mathrm{C} 1$ and $\mathrm{C} 3$ is the jet that is seen at 23:12-23:13 by $\mathrm{C} 3$ and at 23:13-23:14 by $\mathrm{C} 1$. The ion flow in this jet is aligned with the vector from $\mathrm{C} 3$ to $\mathrm{C} 1$, and closer analysis of current sheet orientations, wave activity etc. (not shown) supports the interpretation that it is the same structure that is observed consecutively by $\mathrm{C} 3$ and $\mathrm{C} 1$. The structure seems to expand somewhat on its way from $\mathrm{C} 3$ to $\mathrm{C} 1$, which explains the longer duration of the observation at $\mathrm{C} 1$. This supports the view that the flow velocity variations recorded during the event represent true temporal variations, not spatial ones.

Our conclusion on event 2 is that we observe large shear angle reconnection which is patchy and non-continuous. On larger scales, however, observed from farther away, this reconnection might appear continuous or quasi-continuous, as we do not see any long quiet intervals during the crossing. The timescale of the temporal variations is approximately a minute, consistent with the timescale of the mirror mode fluctuations immediately outside the magnetopause.

\section{Discussion}

In both events the timescale of the plasma jet variations at the magnetopause matches the timescale of the mirror mode fluctuations that convect toward the magnetopause. This is very suggestive and good circumstancial evidence in favour of causal connection, but not sufficient to prove it. Ideally one would want to be able to observe simultaneously both the inflow and outflow regions of an $\mathrm{x}$-line and then to associate certain variations in jet speed one-to-one with corresponding fluctuations in inflowing plasma. However, this would require a very fortuitous spacecraft configuration, which we did not find. It is also questionable whether the modulation would be so straightforward that one could even in principle recognise the effects of individual fluctuations.

Having presented our observations, we turn back to the theoretical estimate (Eq. 2) from Sect. 2 and put in real numbers. For event 1, the magnetosphere has $B_{1}=75 \mathrm{nT}$ and the magnetosheath plasma between the mirror mode magnetic dips has $B_{2}=50 \mathrm{nT}, \rho_{2}=25 \mathrm{~cm}^{-3}$. This gives predicted reconnection jet speed of $345 \mathrm{~km} \mathrm{~s}^{-1}$, which is in excellent agreement with observations: it is just the value at which most fast jets in Fig. 3 peak. The jet recorded by $\mathrm{C} 1$ just before 05:08 stands out as an exception, showing a higher speed. Representative numbers for the bottoms of the mirror mode magnetic dips are $B_{2}=25 \mathrm{nT}, \rho_{2}=35 \mathrm{~cm}^{-3}$. These numbers in Eq. (2) lead to $v_{\text {out }}=184 \mathrm{~km} \mathrm{~s}^{-1}$. A jet of this speed would in practice be indistinguishable from the ambient sheath flow. Thus continuous, inherently steady reconnection modulated by the changing properties of the magnetosheath side inflow would produce apparently bursty outflow. This pseudo-bursty reconnection could explain the observations of event 1 .

For event 2, the result of Eq. (2) is shown in the last panel of Fig. 1. It ranges from $200 \mathrm{~km} \mathrm{~s}^{-1}$ for slow outflow periods to $400 \mathrm{~km} \mathrm{~s}^{-1}$ for top jet speeds. The observed speeds are lower, most jets peaking at about $200 \mathrm{~km} \mathrm{~s}^{-1}$ and the fastest one at $290 \mathrm{~km} \mathrm{~s}^{-1}$. One possible reason to this is that Eq. (2) was derived for antiparallel reconnection. In event 2 the angle between the reconnecting fields was about $140^{\circ}$, which already implies a notable guide field. The effects of a guide field are not accounted for in Eq. (2). However, the derivation of Eq. (2) by Cassak and Shay (2007) was based on energy conservation principles, and the relative changes between the amount of available magnetic energy and energy required to accelerate the inflowing plasma remain even in the non-antiparallel case. Thus Eq. (2) is applicable as an order-of-magnitude scaling law, but it should not be interpreted as an accurate prediction. The observed jet speeds exhibit variation similar to that given by the scaling law. But when we remember also the flow reversals of event 2 , it is clear that modulated continuous reconnection at one stable $\mathrm{x}$ line, which was sufficient to explain event 1 , cannot explain event 2. Reconnection most likely occurs in true bursts at several locations on an $\mathrm{x}$-line. The process is thus inherently unstable, and the magnetic humps and dips of mirror modes may serve to trigger the onset and cessation of reconnection bursts. We do not rule out spatially patchy reconnection even for event 1 , but only for event 2 do we have evidence for it.

Reconnection can be unstable even under steady boundary conditions. For example, Ding et al. (1992) performed particle simulations where periods of quasi-steady single $\mathrm{X}$-line reconnection and intermittent multiple X-line reconnection occurred even in the same run. More recently, using a kinetic simulation, Karimabadi et al. (2007) found that unstable expansion of the electron diffusion region can lead to variable reconnection rate at a single $\mathrm{X}$-line. On the other hand, the particle-in-cell simulations of asymmetric current sheets by Pritchett (2008) exhibited stable reconnection rate. When upstream boundary conditions are unsteady due to mirror modes or other fluctuations, the effect on reconnection rate and outflow velocity may not be as simple as the scaling laws derived by Cassak and Shay (2007) suggest. An intricate interplay between external fluctuations and the intrinsic instability of the reconnection process can be expected, as may 
happen in our event 2. To determine exactly what role the fluctuations in inflow play in the observed variations would require simultaneous multi-scale observations of the inflow and the diffusion region.

\section{Conclusions}

We have presented observations of two events of non-steady reconnection on the subsolar magnetopause. In both cases the reconnection process shows variations on a timescale of the order of one minute. Mirror mode fluctuations appear as strong magnetic fluctuations on the same timescale as the magnetosheath plasma flows toward the magnetopause. We showed that modulation of reconnection outflow velocity by mirror modes is a theoretically sufficient explanation and, given the coincidence of timescales, also a likely explanation for the non-steadiness of reconnection in the first of our two events. In the second event reconnection is patchy and thus inherently unstable, and mirror modes may play a role in triggering the variations.

Acknowledgements. We thank N. Omidi for discussios on possibilities to simulate the phenomenon and $\mathrm{K}$. Trattner for modelling the expected global reconnection line location for the events.

Topical Editor I. A. Daglis thanks S. W. H. Cowley and two other anonymous referees for their help in evaluating this paper.

\section{References}

Balogh, A., Carr, C. M., Acuña, M. H., Dunlop, M. W., Beek, T. J., Brown, P., Fornacon, K.-H., Georgescu, E., Glassmeier, K.H., Harris, J., Musmann, G., Oddy, T., and Schwingenschuh, K.: The Cluster Magnetic Field Investigation: overview of in-flight performance and initial results, Ann. Geophys., 19, 1207-1217, doi:10.5194/angeo-19-1207-2001, 2001.

Cassak, P. A. and Shay, M. A.: Scaling of asymmetric magnetic reconnection: General theory and collisional simulations, Phys. Plasmas, 14, 102114, doi:10.1063/1.2795630, 2007.

Coleman, I. J.: A multi-spacecraft survey of magnetic field line draping in the dayside magnetosheath, Ann. Geophys., 23, 885900, doi:10.5194/angeo-23-885-2005, 2005.

Cowley, S. W. H.: The causes of convection in the Earth's magnetosphere: A review of developments during the IMS, Rev. Geophys., 20(3), 531-565, 1982.

Ding, D. Q., Lee, L. C., and Kennel, C. F.: The beta dependence of the collisionless tearing instability at the dayside magnetopause, J. Geophys. Res., 97(A6), 8257-8267, 1992.

Dungey, J. W.: Interplanetary magnetic field and the auroral zones, Phys. Rev. Lett., 6, 47-48, 1961.

Escoubet, C. P., Fehringer, M., and Goldstein, M.: Introduction: The Cluster mission, Ann. Geophys., 19, 1197-1200, doi:10.5194/angeo-19-1197-2001, 2001.

Génot, V., Budnik, E., Hellinger, P., Passot, T., Belmont, G., Trávníček, P. M., Sulem, P.-L., Lucek, E., and Dandouras, I.: Mirror structures above and below the linear instability threshold: Cluster observations, fluid model and hybrid simulations,
Ann. Geophys., 27, 601-615, doi:10.5194/angeo-27-601-2009, 2009.

Hasegawa, A: Drift mirror instability in the magnetosphere, Phys. Fluids, 12, 2642-2650, 1969.

Joy, S. P., Kivelson, M. G., Walker, R. J., Khurana, K. K., Russell, C. T., and Paterson, W. R.: Mirror mode structures in the Jovian magnetosheath, J. Geophys. Res., 111, A12212, doi:10.1029/2006JA011985, 2006.

Karimabadi, H., Daughton, W., and Scudder, J.: Multi-scale structure of the electron diffusion region, Geophys. Res. Lett., 34, L13104, doi:10.1029/2007GL030306, 2007.

Kawano, H. and Russell, C. T.: Dual-satellite observations of the motions of flux transfer events: Statistical analysis with ISEE 1 and ISEE 2, J. Geophys. Res., 110, A07217, doi:10.1029/2004JA010821, 2005.

Khrabrov, A. V. and Sonnerup, B. U. Ö.: DeHoffman-Teller analysis, in: Analysis methods for multi-spacecraft data, edited by: Paschmann, G. and Daly, P. W., ISSI scientific report SR-001, 1998.

$\mathrm{Ku}$, H. C. and Sibeck, D. G.: Flux transfer events produced by bursty merging at a single X line, J. Geophys. Res., 103, 1496514978, 1998.

Laitinen, T. V., Janhunen, P., Pulkkinen, T. I., Palmroth, M., and Koskinen, H. E. J.: On the characterization of magnetic reconnection in global MHD simulations, Ann. Geophys., 24, 30593069, doi:10.5194/angeo-24-3059-2006, 2006.

Laitinen, T. V., Palmroth, M., Pulkkinen, T. I., Janhunen, P., and Koskinen, H. E. J.: Continuous reconnection line and pressure-dependent energy conversion on the magnetopause in a global MHD model, J. Geophys. Res., 112, A11201, doi:10.1029/2007JA012352, 2007.

Lockwood, M., Lanchester, B. S., Frey, H. U., Throp, K., Morley, S. K., Milan, S. E., and Lester, M.: IMF control of cusp proton emission intensity and dayside convection: implications for component and anti-parallel reconnection, Ann. Geophys., 21, 955-982, doi:10.5194/angeo-21-955-2003, 2003.

Lucek, E. A., Dunlop, M. W., Horbury, T. S., Balogh, A., Brown, P., Cargill, P., Carr, C., Fornaon, K.-H., Georgescu, E., and Oddy, T.: Cluster magnetic field observations in the magnetosheath: four-point measurements of mirror structures, Ann. Geophys., 19, 1421-1428, doi:10.5194/angeo-19-1421-2001, 2001.

Pedersen, A., Lybekk, B., André, M., et al.: Electron density estimations derived from spacecraft potential measurements on Cluster in tenuous plasma regions, J. Geophys. Res., 113, A07S33, doi:10.1029/2007JA012636, 2008.

Phan, T. D., Hasegava, M., Øieroset, M., Mukai, T., Lin, R. P., and Paterson, W.: Simultaneous Geotail and Wind observations of reconnection at the subsolar and tail flank magnetopause, Geophys. Res. Lett., 33, L09104, 2006.

Pritchett, P. L.: Collisionless magnetic reconnection in an asymmetric current sheet, J. Geophys. Res., 113, A06210, doi:10.1029/2007JA012930, 2008.

Rème, H., Aoustin, C., Bosqued, J. M., et al.: First multispacecraft ion measurements in and near the Earth's magnetosphere with the identical Cluster ion spectrometry (CIS) experiment, Ann. Geophys., 19, 1303-1354, doi:10.5194/angeo-19-1303-2001, 2001.

Retinò, A., Vaivads, A., André, M., Sahraoui, M., Khotyaintsev, Y., Pickett, J. S., Bavassano Cattaneo, M. B., Marcucci, M. F., Morooka, M., Owen, C. J., Buchert, S. C., and Cornilleau-Wehrlin, 
N.: Structure of the separatrix region close to a magnetic reconnection X-line: Cluster observations, Geophys. Res. Lett., 33, L06101, doi:10.1029/2005GL024650, 2006.

Russell, C. T. and Elphic, R. C.: ISEE observations of flux transfer events at the dayside magnetopause, Geophys. Res. Lett., 6(1), 33-36, 1979.

Sonnerup, B. U. Ö., Hasegawa, H., and Paschmann, G.: Anatomy of a flux transfer event seen by Cluster, Geophys. Res. Lett., 31, L11803, doi:10.1029/2004GL020134, 2004.

Soucek, J., Lucek, E., and Dandouras, I.: Properties of magnetosheath mirror modes observed by Cluster and their response to changes in plasma parameters, J. Geophys. Res., 113, A04203, doi:10.1029/2007JA012649, 2008.

Tátrallyay, M., and Erdős, G.: The evolution of mirror mode fluctuations in the terrestrial magnetosheath, Planet. Space Sci., 50, 593-599, 2002.

Trattner, K. J., Mulcock, J. S., Petrinec, S. M., and Fuselier, S. A.: Probing the boundary between antiparallel and component reconnction during southward interplanetary magnetic field conditions, J. Geophys. Res., 112, A08210, doi:10.1029/2007JA012270, 2007.
Treumann, R. A., Jaroschek, C. H., Constantinescu, O. D., Nakamura, R., Pokhotelov, O. A., and Georgescu, E.: The strange physics of low frequency mirror mode turbulence in the high temperature plasma of the magnetosheath, Nonlin. Processes Geophys., 11, 647-657, doi:10.5194/npg-11-647-2004, 2004.

Tsurutani, B. T., Smith, E. J., Anderson, R. R., Ogilvie, K. W., Scudder, J. D., Baker, D. N., and Bame, S. J.: Lion roars and nonoscillatory drift mirror waves in the magnetosheath, J. Geophys. Res., 87(A8), 6060-6072, 1982.

Tsyganenko, N. A.: Modeling the Earth's magnetospheric magnetic field confined within a realistic magnetopause, J. Geophys. Res., 100(A4), 5599-5612, 1995.

Wang, Y. L., Elphic, R. C., Lavraud, B., Taylor, M. G. G. T., Birn, J., Russell, C. T., Raeder, J., Kawano, H., and Zhang, X. X.: Dependence of flux transfer events on solar wind conditions from 3 years of Cluster observations, J. Geophys. Res., 111, A04224, doi:10.1029/2005JA011342, 2006. 